\title{
Die femme fatale im Kirchenstaat: Positionen der Querelle des Femmes in Rom (1622-1678)
}

Als Silvio Antoniano, der Sekretär des später heiliggesprochenen Carlo Borromeo, 1588 einen Traktat über christliche Kindererziehung verfasste, umschrieb er die Kompetenzen eines Fräuleins aus gutem Hause wie folgt: »Man erwartet von einem Mädchen aus guter Familie, dass es das Marienoffizium und dasjenige der Heiligen aufsagen kann, und im übrigen soll es spinnen und nähen und sich mit anderen weiblichen Tätigkeiten befassen. ${ }^{1}$ Von intellektuellen Aktivitäten riet Antoniano ausdrücklich ab, denn dies könne die von Natur aus schon eitlen Frauen erst recht hochmütig machen. ${ }^{2}$

Etwa hundert Jahre später scheint Antonianos Ideal der Frauenbildung immer noch Gültigkeit besessen zu haben. In dem 1675 in Rom erschienenen Benimmbuch Il Cavaliere e la Dama wurden nach wie vor Handarbeiten als die optimale weibliche Beschäftigung propagiert: »Alle Autoren, die den Lebensstil der Damen und gar der Prinzessinnen und Königinnen behandeln, loben es als vorbildlich, dass sie Müßiggang vermeiden, indem sie zu Hause zusammen mit ihren Dienerinnen und ihrem Gefolge die weiblichen Fertigkeiten ausüben und etwa Leinen, Wolle, Seide und andere Dinge dieser Art bearbeiten. $\aleph^{3}$ Dass die römische Nobilität jenem Ideal zu folgen suchte, wird eindrucksvoll durch eine Nachricht von 1683 illustriert, der zufolge die Principessa Borghese mit über 60 Frauen ihres Gefolges Livreen und Prunkgewänder bestickte. $^{4}$

Wie etliche dokumentbasierte Studien gezeigt haben, beschränkten sich die römischen Damen aber keineswegs nur aufs Handarbeiten, sondern besaBen teilweise recht beachtliche Handlungsspielräume. Sie unterstützten ihre Ehemänner durch soziale Kontaktpflege und informelle Diplomatie, beteiligten sich an der Erziehung ihrer Kinder, förderten deren Karrieren und Eheprojekte, übernahmen innerhalb ihrer Familie wichtige administrative und karitative Aufgaben, protegierten religiöse Orden und taten sich vor allem auf religiösem Gebiet auch als Bauherrinnen hervor. ${ }^{5}$

Eine besonders herausgehobene Position besaßen die weiblichen Verwandten des Papstes, beispielsweise Anna Colonna Barberini. Anna Colonna, die aus einer der vornehmsten Adelsfamilien Roms stammte, war 1627 mit Taddeo 
Barberini, einem Neffen Urbans VIII., verheiratet worden. Simona Feci und Maria Antonietta Visceglia haben detailliert dargelegt, wie Anna ihren Einfluss geltend machte, um zur Klientelbildung beizutragen und die Interessen ihrer beiden Familien zu fördern. Die Autorinnen gelangten zu der Schlussfolgerung, dass Annas Gestaltungsmöglichkeiten dennoch relativ begrenzt waren: Nur niederrangige Bittsteller suchten ihre vermittelnde Fürsprache, während sich mächtigere Personen direkt an die männlichen Entscheidungsträger wandten. ${ }^{6}$

Als die Barberini 1646 ins Exil flohen, musste Anna in Rom zurückbleiben, um dort die Interessen der Familie zu wahren. ${ }^{7}$ Als Frau durfte sie jedoch nicht persönlich vor dem Senat erscheinen; vielmehr musste sie in der kapitolinischen Kirche Santa Maria in Aracoeli warten, während ihre Bittschrift von einem männlichen Verwandten dem Senat vorgetragen wurde. ${ }^{8}$ Die Konservatoren wiesen Annas Argumente zurück, vermerkten aber immerhin respektvoll, dass sie durch ihre soziale Stellung zu mehr Einsicht befähigt sei als die »gewöhnliche Schar der Frauen«. Die Formulierung »donna di grand'intendimento e nata fuori della vulgare mescolanza del femíneo stuolo « (»Frau von großer Einsicht, durch ihre Geburt erhoben über das vulgäre Gemisch der Frauenschar «) lässt allerdings erkennen, wie wenig die Konservatoren im allgemeinen von Frauen hielten. ${ }^{9}$ Kardinal Girolamo Colonna distanzierte sich von der Initiative seiner Schwester Anna, indem er ihr öffentlich »sciocchezza« (Dummheit) vorwarf. ${ }^{10}$

Die misogyne Grundhaltung, die sowohl in den Worten des Kardinals als auch in denen der Konservatoren schlaglichtartig aufblitzt, hatte schon seit dem 14. Jahrhundert die sogenannte Querelle des Femmes befeuert. Die sowohl in Frankreich als auch in Italien mit großer Vehemenz geführte Debatte betraf die Fähigkeiten und die gesellschaftliche Stellung der Frauen. Die Etikettierung der Debatte als Querelle des Femmes ist modernen Ursprungs, besitzt aber durchaus historische Berechtigung, denn Martin Le Franc sprach in seinem 1440-42 erschienenen Champion des Dames bereits explizit von einer »querelle des dames «. ${ }^{11}$ Das Streitgespräch ist allerdings auch unter vielen anderen Namen geführt worden (als controverses, débat, discours, apologie, défense etc.), d. h. es gab in der betreffenden Epoche noch keinen allgemein akzeptierten Oberbegriff für die »Frauenfrage «. ${ }^{12}$ Martin Le Franc verwendete »querelle « im Sinne von "Anklage« oder »Prozess«; der Champion des Dames ist ein »Verteidiger der edlen Frauen«, die sich über die Verunglimpfung ihres Geschlechts durch die Männer beschwert haben. Der Begriff "Querelle des Femmes« bedeutet also einerseits Wehklage und Anklage seitens der Frauen, andererseits aber auch »Disput der Frauen « oder "Streit um die Frauen ${ }^{13}$

Während das 16. Jahrhundert als die eigentliche Blütezeit der Streitschriften von und über Frauen zu gelten hat, sollen im Kontext des vorliegenden Sammelbandes zwei relativ späte Publikationen in den Blick genommen werden, die beide speziell für die Situation der Frauen in Rom aufschlussreich sind. Die im Titel genannten Eckdaten 1622 bzw. 1678 beziehen sich auf Cristofano 
Bronzinis Dialog Della dignità, E nobiltà delle donne, der in vier Bänden zwischen 1622/24 und 1632 erschien, sowie auf die 1678 veröffentlichte Apologia der Maria Mancini. Diese Werke thematisieren zwei verschiedene Aspekte weiblicher Freiheit: zum einen die politische Mitbestimmung, zum anderen die persönliche Selbstbestimmung. Es wird im Folgenden aber nicht nur um die genannten Texte gehen, sondern vor allem auch um die bereits angeschnittene Frage, welche Handlungsspielräume Frauen im Rom des Seicento besaßen und inwiefern Kunstwerke zur Konstruktion weiblicher Rollenbilder beitrugen.

\section{Della dignità, \& nobiltà delle donne von Cristofano Bronzini: ein Auftragswerk Christianes von Lothringen?}

Bronzinis Dialog wurde zwar in Florenz verlegt, doch spielt er in Rom - laut Einleitungstext im Garten der Villa Medici auf dem Pincio. Insbesondere in Rom sorgte das Werk für Furore, denn während der Florentiner Inquisitor keinerlei Beanstandungen gehabt hatte, verbot die römische Indexkongregation 1622 die Veröffentlichung des ersten Bandes und ließ diesen erst 1624 in einer korrigierten Fassung erscheinen. Xenia von Tippelskirch hat dargelegt, welche Eingriffe die Zensoren verlangten. ${ }^{14}$ Bevor ich auf die Frage eingehe, was diese erzwungenen Korrekturen über das römische Frauenbild aussagen, möchte ich zunächst die politische Bedeutung von Bronzinis Text herausstellen.

Als die ersten Bände von Bronzinis Dialog herauskamen, hatten in Florenz ungewöhnlicherweise zwei Frauen die Regierungsgewalt inne: Christiane von Lothringen und ihre Schwiegertochter Maria Magdalena von Österreich. Von 1621 bis 1628 agierten sie als Vormünder des minderjährigen Großherzogs Ferdinando II. de' Medici, der Maria Magdalenas Sohn und Christianes Enkel war. ${ }^{15}$ Da Bronzini die ersten beiden Bände seines Werks Maria Magdalena von Österreich widmete, konzentrierte sich Xenia von Tippelskirchs Interpretation auf sie. Die jüngere der beiden Regentinnen führte den Titel einer Erzherzogin von Innerösterreich und konnte dadurch den Vorrang vor ihrer Schwiegermutter Christiane von Lothringen beanspruchen, die »nur« Großherzogin war. Zudem war Maria Magdalena die Schwester des Kaisers, was ihr mehr politisches Gewicht verlieh. Deswegen stand sie während der Regentschaft im Vordergrund. Um die Intentionen von Bronzinis Text klarer zu konturieren, soll im Folgenden jedoch gezeigt werden, dass die Großherzogin Christiane von Lothringen die treibende Kraft hinter dem Publikationsprojekt war.

Im vierten Band des Dialogs findet sich ein Porträt Bronzinis, das ihn klar als »Medici-Kreatur « charakterisiert (Abb. 1). ${ }^{16}$ Über seinem Kopf prangt das Medici-Wappen mit den sechs palle (Kugeln), auf die sich die darunter angebrachte Inschrift bezieht: »Più nella mente impresse ("noch stärker in den Geist eingeprägt «). ${ }^{17}$ Die Medici-Zeichen wurden demnach nicht nur in die Kupferplatte des Stechers, sondern noch mehr in den Geist Bronzinis eingra- 


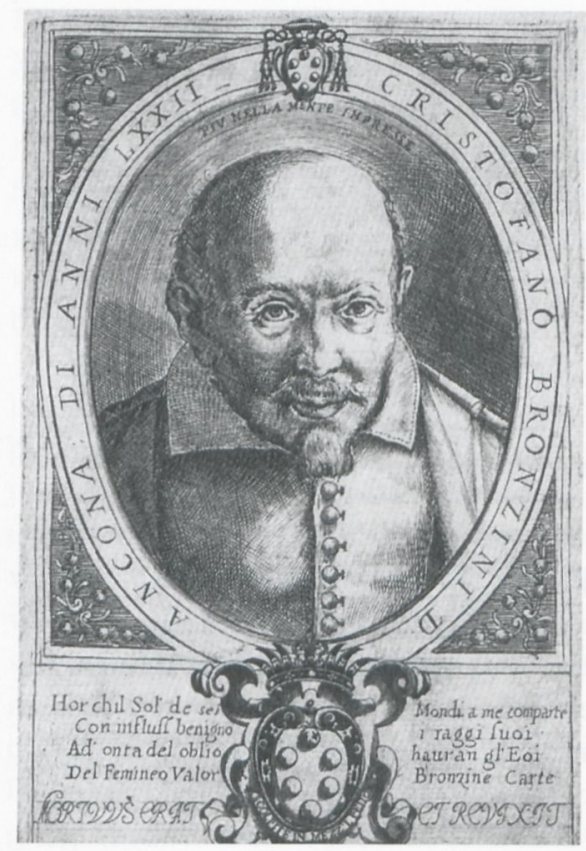

1. Anonymer Künstler: Cristofano Bronzini d'Ancona, Kupferstich aus: Della Virtù, e Valore delle Donne Illustri. Dialogo di Christofano Bronzini d'Ancona. Diviso in quattro Settimane; e ciascheduna di esse in sei Giornate. Settimana Seconda, Giornata Settima, Florenz 1632. Florenz, Biblioteca Nazionale Centrale

viert. Wohl nicht zufällig ist dieses Porträt gerade jenem Band des Dialogs vorangestellt, der eine Widmung an Christiane von Lothringen trägt - war sie doch diejenige, die Bronzini an den Medici-Hof geholt hatte. Die Kontakte zwischen Bronzini und Christiane reichen bis in das Jahr 1610 zurück, in dem Maria Magdalena von Österreich gerade erst als junge Braut nach Florenz gekommen war und politisch noch keine nennenswerte Rolle spielte. Zu jener Zeit dominierte Christiane von Lothringen die Florentiner Politik, da ihr Ehemann Ferdinando I. 1609 verstorben war und ihr noch sehr junger Sohn Cosimo II. bei allen Entscheidungen ihren Rat einholte. ${ }^{18} 1610$ bemühte sie sich darum, ihren Sohn Francesco zum Kardinal zu machen, und warb in diesem Zusammenhang Bronzini als dessen Berater an. Als später nicht Francesco, sondern dessen Bruder Carlo Kardinal wurde, zählte Bronzini zu seinem Gefolge und assistierte ihm im Konklave von 1621, aus dem Bronzini der Großherzogin berichtete. ${ }^{19}$

In den Jahren, in denen Bronzini seinen Dialog verfasste, stand er also in einem Klientelverhältnis zu Christiane von Lothringen und ihrem Sohn Carlo. Darüber hinaus hatte die Lothringerin bereits zuvor ein deutliches Interesse an der Querelle des Femmes bekundet. Sie war am Hof ihrer Großmutter, der französischen Königin Caterina de' Medici, aufgewachsen und hatte schon in Frankreich miterlebt, wie sich die Querelle politisch instrumentalisieren ließs. Da Caterina de' Medici starken Einfluss auf die französische Politik nahm, war sie vielfältigen Attacken ausgesetzt, die auch ganz generell die Regie- 
rungsunfähigkeit von Frauen behaupteten. ${ }^{20}$ Gerade angesichts solcher misogyner Anwürfe entstanden im Umfeld der Königin mehrere literarische Werke, die die besonderen Qualitäten und Fähigkeiten von Frauen betonten. Marie de Romieus Brief discours: Que l'excellence de la femme surpasse celle de l'homme suchte 1581 nicht nur die Überlegenheit der Frauen zu beweisen, sondern ehrte auch die gelehrten weiblichen Mitglieder des Königshauses als illustre Humanistinnen. ${ }^{21}$ Caterinas Tochter, die zeitweilige Königin Margarete von Valois, verfasste einen kurzen Traktat, der ebenfalls den Vorrang des weiblichen Geschlechts postulierte. ${ }^{22}$ Es war jedoch Matteo Zampini vorbehalten, die Würdigung der französischen Gynäkokratie auf die Spitze zu treiben: Sein Elogio della gran Caterina di Medici kam 1586 gleich in vier Sprachen heraus - mit einer Widmung an Christiane von Lothringen. ${ }^{23}$

Als Großherzogin der Toskana nutzte Christiane ebenfalls literarische Werke, um ihre politische Position als designierte Regentin zu festigen. 1596 erschien in Florenz eine erweiterte italienische Neuausgabe von Boccaccios De claris mulieribus, eines Schlüsseltexts der Querelle des Femmes, mit einer Widmung an Christiane von Lothringen. Der Herausgeber Francesco Serdonati fügte Boccaccios Katalog illustrer Frauen etliche weitere Exempel hinzu, darunter die toskanischen (Groß)herzoginnen ebenso wie die lothringischen Vorfahrinnen Christianes. ${ }^{24}$ Besonders großen Raum beanspruchten die Lebensbeschreibungen von Caterina de' Medici und - als krönender Abschluss des gesamten Bandes - Christianes von Lothringen. ${ }^{25}$ Ausführlich betonte Serdonati, die französische Königin habe Christiane in alle Geheimnisse ihrer Regierungskunst eingeweiht. ${ }^{26}$ In einem vorangestellten discorso »Über die weibliche Vollkommenheit« vertrat Serdonati wortreich die Ansicht, dass Männer und Frauen über gleiche Fähigkeiten verfügen. ${ }^{27}$

Sobald Christiane von Lothringen und Maria Magdalena von Österreich jedoch die Regentschaft übernahmen, sahen sie sich vielfältigen misogynen Vorbehalten ausgesetzt. Dies geht etwa aus einem Tacitus-Kommentar hervor, den Virgilio Malvezzi 1622 dem minderjährigen Großherzog Ferdinando II. widmete: »Es gibt nichts, was von den Männern mehr gehasst und verabscheut wird, als einer Frau dienen zu müssen, denn dies widerspricht der Vernunft und Gottes Geboten, vor allem aber den Gesetzen der Natur [...], besitzt doch jenes Geschlecht (nicht immer, aber meistens) weder Klugheit noch Tugend; stattdessen viel Hochmut und ebensoviel Lüsternheit, ist also völlig ungeeignet zum Regieren. $\ll^{28}$

Ebenfalls 1622, ein Jahr nach Beginn der Florentiner Regentschaft, erschien der erste Band von Cristofano Bronzinis Dialog Della dignità, E nobiltà delle donne. Zweifellos handelte es sich um den Versuch, die Querelle des Fermmes erneut politisch zu instrumentalisieren, um die Regierungskompetenz der Frauen zu unterstreichen. Angesichts der lang etablierten Kontakte zwischen Bronzini und Christiane von Lothringen sowie in Anbetracht ihres vorangehenden Engagements auf dem Gebiet der Querelle des Fermmes ist davon auszugehen, dass sie Bronzinis Publikationsprojekt initiierte. 
Wie bei Zampini und Serdonati wurden wiederum Caterina de' Medici und Christiane von Lothringen prominent in Szene gesetzt. Während Bronzini Maria Magdalena von Österreich hauptsächlich für ihre Frömmigkeit und ihre Reitkünste lobte, präsentierte er Christiane als eine überaus geschickte Politikerin, die explizit sowohl Frauen als auch Männern als Vorbild dienen könne. ${ }^{29}$ Die detailreiche, insgesamt 15 Seiten umfassende Würdigung Christianes geht weit über das hinaus, was bisher in der italienischen QuerelleLiteratur üblich war. ${ }^{30}$ Während in Rom eine Frau wie Anna Colonna trotz ihres hohen Rangs noch nicht einmal persönlich vor dem Senat erscheinen durfte, führte Bronzini seiner Leserschaft eindringlich vor Augen, wie Frauen in Florenz durchaus Regierungsverantwortung übernehmen konnten und welcher Qualitäten es dafür bedurfte.

Warum nun spielt Bronzinis Dialog in Rom? Wie sich den vorangestellten »Regieanweisungen« entnehmen lässt, haben sich im Garten der römischen Villa Medici vornehme Gesprächsteilnehmer aus verschiedenen Staaten eingefunden: die Römerin Margherita, die Mantuanerin Vittoria, die Florentinerin Leonora, der nicht namentlich bezeichnete »Principe der Toskana, Tolomei aus Ferrara und Cristofano Bronzini aus Ancona. Die fingierte Unabhängigkeit der meisten Dialogpartner vom Florentiner Hof und die räumliche Distanz zu Florenz lassen das Gesagte objektiver wirken. Es erscheint nicht als höfische Panegyrik, sondern als sachlicher Austausch von Argumenten, wobei durch den Frauenfeind Tolomei auch konträre Positionen zu Wort kommen dürfen.

Trotz dieser recht diplomatischen diskursiven Form provozierte der Text in Rom ein empörtes Echo. Bronzini suchte die Überlegenheit der Frauen über die Männer nachzuweisen. Dass der Mann, der der Frau eigentlich dienen solle, sich zum Herrn aufgeschwungen habe, sei "ungerecht und tyrannisch", denn der Mann sei im Vergleich zur Frau nur so viel wert wie der einzelne Stein in Relation zu einem ganzen Haus. Solche und ähnliche Aussagen, die gesellschaftlich destabilisierend wirken konnten, alarmierten die Inquisition und führten zur Streichung bzw. Abmilderung der inkriminierten Passagen. ${ }^{31}$

Ferner konnten die Kardinäle nicht die Ansicht tolerieren, Eva sei die Krone der Schöpfung gewesen. Entsprechend wurde folgende Aussage zensiert: »dass in der Frau, der würdigsten aller Kreaturen, alles Wissen und Können des Schöpfers seinen Endpunkt gefunden habe, wonach es Gott nicht möglich war, ein hervorragenderes oder würdigeres Wesen zu erschaffen, da nichts über die Frau Hinausgehendes zu finden oder vorzustellen ist. $\ll^{32}$ Beanstandet wurde insbesondere aber die daraus abgeleitete Ansicht, »dass die Frau von Gott zur Herrin über den Mann erschaffen worden sei, und der Mann ihr untertan, um ihr zu dienen, wenngleich sie dann durch den Sündenfall ihm untergeordnet wurde $\ll{ }^{33}$ Diese letztlich politisch motivierte Argumentation, Frauen seien zum Herrschen (und somit auch zum Regieren) befähigt, wurde nicht nur von dem bereits zitierten Virgilio Malvezzi, sondern auch von der römischen Gesellschaft scharf zurückgewiesen. 


\section{Die femme fatale im Palazzo Chigi: eine Antwort auf Bronzini?}

Geradezu wie eine Antwort auf die genannten umstrittenen Passagen wirkt das Freskenprogramm, das Kardinal Giovan Battista Deti zwischen 1625 und 1627 in der Galerie seines römischen Stadtpalastes (heute Palazzo Chigi) ausführen ließ. Deti war 1599 im Alter von 17 Jahren durch seinen mächtigen Verwandten Papst Clemens VIII. Aldobrandini zum Kardinal erhoben worden und füllte sein Amt mit entsprechend geringer religiöser Überzeugung aus; vielmehr stand er in dem Ruf, allen Arten von Lastern und Ausschweifungen verfallen zu sein. ${ }^{34}$ Als Thema der Galerieausmalung wählte er biblische Frauengestalten. Damit knüpfte er an eine familiäre Tradition an, denn auch Kardinal Pietro Aldobrandini hatte seine Villa in Frascati mit ähnlichen Szenen ausstatten lassen. ${ }^{35}$ Deti setzte durch die Auswahl der dargestellten Episoden jedoch neue Akzente.

Flaminio Allegrini, den Deti mit der Ausmalung seiner Galerie beauftragte, war ein Schüler jenes Giuseppe Cesari (Cavalier d'Arpino), der die Fresken in Frascati geschaffen hatte. ${ }^{36}$ Der Bezug zwischen beiden Bildprogrammen ist evident, denn sowohl in der Galerie der Villa Aldobrandini als auch in jener des Palazzo Chigi wird das Gewölbe von drei Szenen aus dem Leben der ersten Menschen dominiert. Die beiden seitlichen Fresken vergegenwärtigen jeweils den Sündenfall und die Vertreibung aus dem Paradies. Das große Mittelbild zeigt in Frascati die Erschaffung Adams, in der Galleria Deti die Erschaffung Evas. ${ }^{37}$ Eva ist in der Galleria Deti klar die Protagonistin des Geschehens, denn sie nimmt in allen drei Gemälden die prominenteste Bildposition ein und zieht durch ihr helles Inkarnat die Blicke auf sich. Das Mittelbild (Abb. 2) präsentiert uns Adam in Rückansicht, so dass sein Antlitz verborgen bleibt. Eva bildet den erotischen Blickfang des Gemäldes. Sie wendet sich hingebungsvoll ihrem Schöpfer zu, der sie segnet. Gestik und Haltung dieser beiden Figuren drücken Zuneigung und Harmonie aus. Der Pfau am linken Bildrand spiegelt Evas körperliche Schönheit, dürfte aber auch auf das Laster der Eitelkeit anspielen. Das auf der Mittelachse platzierte Hündchen steht traditionell für die Tugend der Treue und weist somit indirekt darauf hin, dass Eva Gottes Gebot gerade nicht treu befolgte. Bronzinis These, Eva sei als das eigentlich noblere Geschöpf erschaffen worden, wird hier also durch die allusive Vergegenwärtigung ihrer Lasterhaftigkeit abgelehnt, die in den flankierenden Szenen des Sündenfalls und der Vertreibung aus dem Paradies mit aller Deutlichkeit zum Ausdruck kommt.

Während sich die Ausmalung der Galleria Aldobrandini in Frascati auf die Erschaffung Adams, Sündenfall und Vertreibung beschränkte und biblische Helden bzw. Heldinnen (David, Abigail, Jael, Judith) die angrenzenden Zimmer dominieren, ${ }^{38}$ fasst die Galleria Deti gewissermaßen beide Bildzyklen zusammen: Die drei Szenen aus dem Leben der ersten Menschen werden von zwölf weiteren Gemälden eingerahmt, die Frauen aus dem Alten Testament zeigen (Abb. 3). ${ }^{39}$ Dabei nehmen Judith und Jael besonders prominente Posi- 


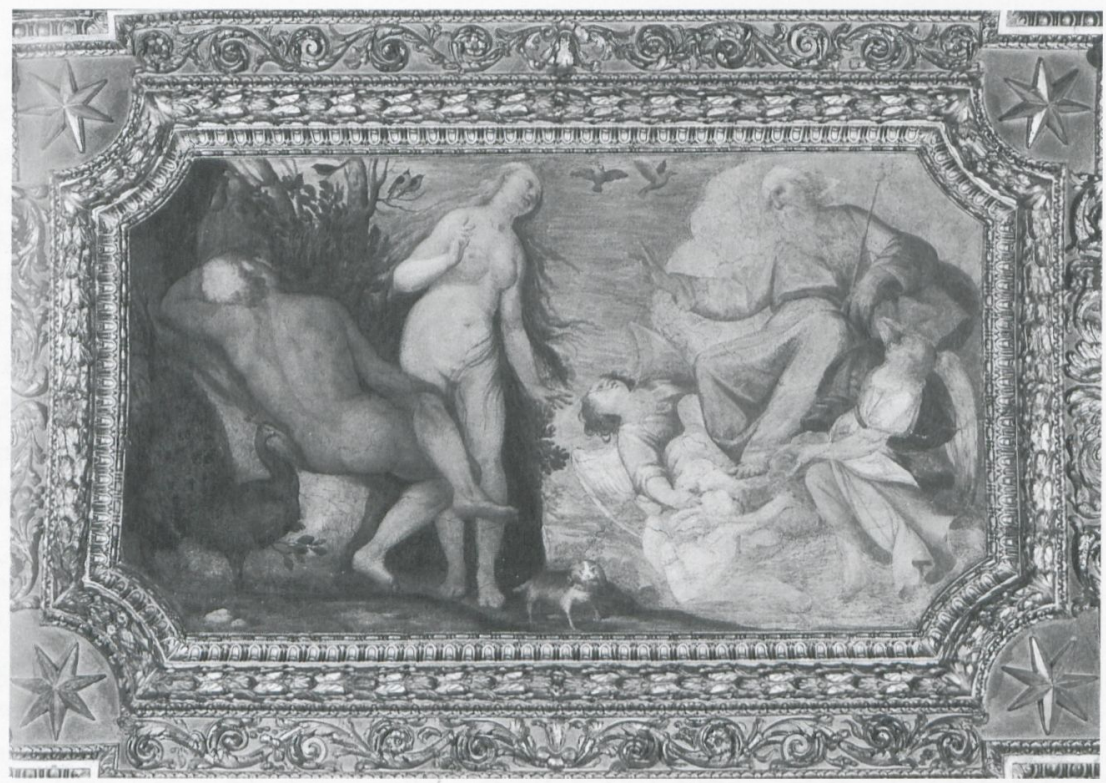

2. Flaminio Allegrini: Die Erschaffung Evas, 1625-1627, Fresko. Rom, Palazzo Chigi, Galleria Deti

tionen ein, da sie die Hauptachse des Gewölbes abschließen. Beide Frauen sind in hochdramatischen Situationen wiedergegeben: Mit dynamischen, weit ausholenden Gebärden schicken sie sich an, ihren jeweiligen männlichen Widersacher zur Strecke zu bringen. Raumgreifend dominieren sie das Bild, während die den Todesstreich erwartenden Männer zu ihren Füßen liegen. ${ }^{40}$

Trotz ihrer Greueltaten galten Judith und Jael als Befreierinnen ihres Volkes. ${ }^{41}$ Durch die Themen, die der Kardinal Deti neu in sein Bildprogramm einfügen ließ, stellte er diese beiden "Heldinnen" hingegen in einen ganz andersartigen Kontext. In den Lünettenbildern der Langseiten geht es stets um Verführung bzw. um sexuelle Gewalt. Beispielsweise ist Tamars Vergewaltigung durch ihren Bruder Amnon dargestellt ${ }^{42}$ sowie die Konkubine des Leviten, die nach einer Gruppenvergewaltigung tot auf der Türschwelle seines Hauses liegt. ${ }^{43}$ Wie Sigrid Epp gezeigt hat, rechtfertigte die zeitgenössische Traktatliteratur das Verhalten der Männer bzw. gab den Frauen eine Mitschuld an diesen grauenhaften Ereignissen. ${ }^{44}$

Da sich das Kardinalskollegium 1622/23 mit Cristofano Bronzinis Traktat Della dignità, e nobiltà delle donne auseinandergesetzt hatte, wirkt die wenig später begonnene Galerie des Kardinals Deti wie die Einladung zu einer Diskussion über die von Bronzini postulierte »Würde und Nobilität der Frauen«. Die ausgewählten Szenen besitzen allesamt eine gewisse Ambivalenz, die dazu herausfordert, das Verhalten der dargestellten Personen kritisch zu reflektieren. ${ }^{45}$ Durch die rings um die zentralen Deckenbilder angeordneten 


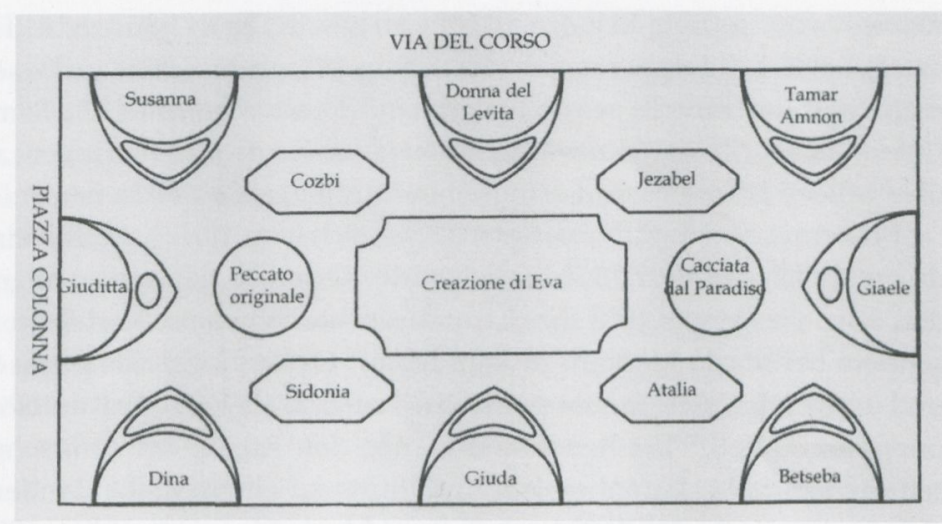

3. Schemazeichnung der Deckendekoration in der Galleria Deti des Palazzo Chigi (nach Epp 2001, S. 87)

alttestamentarischen Frauengestalten gab Deti allerdings eine misogyne Lesweise vor: Die vier Frauen, die durch die begleitenden Inschriften als Sidonia, Athalia, Cozbi und Iezabel identifiziert sind (vgl. Abb. 3), galten als Exempla weiblicher Laster. ${ }^{46}$

Während Bronzini die Ansicht vertrat, die Frau sei ursprünglich zur Herrin über den Mann bestimmt gewesen und sehr wohl zum Regieren befähigt, inszenierte Kardinal Deti einen brutalen Krieg der Geschlechter. In diesem Kontext fungieren die todbringenden Heroinen Judith und Jael quasi als Warnung vor den Gefahren weiblicher Dominanz. Die Akte sexueller Gewalt gegen Frauen verfestigen hingegen die traditionelle Hierarchie der Geschlechter. Das Verhalten der Männer wird gerechtfertigt durch die generelle Lasterhaftigkeit der Frauen, die letztlich aus Evas Ursünde resultiert. Eva erscheint somit als die femme fatale, durch die alles Unheil in die Welt kam. Auf die Verunsicherung, die Bronzinis Thesen auslösten, reagierte Kardinal Deti folglich durch die betonte Affirmation der weiblichen Unterlegenheit.

\section{»Feministische« Positionen in den Memoiren der Maria Mancini}

Während Bronzinis Dialog eine politische Stoßrichtung besitzt, geht es in Maria Mancinis Apologia, ovvero le autentiche memorie di Maria Mancini connestabile Colonna, scritte da lei stessa um individuelle weibliche Selbstbestimmung. Zunächst sei die Autorin kurz vorgestellt. ${ }^{47}$ Maria Mancini entstammte einer römischen Adelsfamilie, wurde aber als Nichte des Kardinals Mazarin vorwiegend am französischen Hof sozialisiert. Dort bewegte sie sich im Kreis der sogenannten précieux und wurde für ihre literarischen Fähigkeiten bewundert. Sie soll die erste große Liebe Ludwigs XIV. gewesen sein, musste 1661 aber aus Gründen der Staatsräson den römischen Adligen Lorenzo Onofrio Colonna heiraten. 1672 verließs sie ihren Mann und flüchtete nach Frankreich, 
wo sie hoffte, Ludwig XIV. erneut für sich gewinnen zu können. Auf Betreiben des Papstes solidarisierte sich der König jedoch mit dem verlassenen Ehemann, der den Eintritt seiner Frau in ein Kloster wünschte. Die Bemühungen Colonnas, Maria Mancini wieder unter seine Kontrolle zu bringen, zogen sich über etliche Jahre hin und erregten in Europa großes Aufsehen. ${ }^{48}$

Mancinis spektakulärer Entschluss, sich von ihrem Mann zu trennen, dürfte durch das Vorbild ihrer Schwester Ortensia (Hortense) angeregt worden sein, die bereits 1668 ihren französischen Ehemann verlassen hatte und seitdem bei Maria Mancini in Rom lebte. ${ }^{49}$ Ortensia galt als große Schönheit und unterstrich dies, indem sie sich in Rom u. a. als Venus mit entblößter Brust porträtieren ließ ${ }^{50}$ Ihr Benehmen war in den Augen der römischen Gesellschaft skandalös, zumal sie ein Verhältnis zu einem nicht standesgemäßen Geliebten unterhielt. ${ }^{51} 1672$ begleitete sie Maria Mancini auf ihrer Flucht nach Frankreich, ließ sich dann aber in Savoyen nieder. Dort verfasste Ortensia ihre Memoiren, die 1675 für großen Wirbel auf dem Buchmarkt sorgten. ${ }^{52}$ Der Erfolg dieser Skandalgeschichte führte dazu, dass 1676 auch eine angebliche Autobiographie Maria Mancinis erschien. Maria selbst distanzierte sich von jenem Werk und antwortete darauf mit ihren "wahren Memoiren«, die 1676 zunächst in französischer Sprache veröffentlicht wurden. 1677 kam eine spanische, 1678 eine italienische und 1679 eine englische Fassung heraus. ${ }^{53}$

Mancinis sogenannte Apologia erzählt nicht nur ihr Leben, sondern versucht auch die Trennung von ihrem Gemahl zu rechtfertigen. Das Verhältnis der Eheleute sei anfangs von großer Zuneigung und Galanterie geprägt gewe$\operatorname{sen}^{54}$ - (eine Behauptung, die ein von Lorenzo Onofrio Colonna in Auftrag gegebenes Rollenporträt des Paares durchaus untermauert, zeichnet er darauf doch in Gestalt des Paris seine Gemahlin als die schönste aller Frauen aus). ${ }^{55}$ Im Laufe der Zeit habe er es jedoch an dem nötigen Respekt vor seiner Gattin fehlen lassen, monierte sie. ${ }^{56}$ Vor allem tadelte sie seine Versuche, ihre Freiheit einzuschränken, indem er ihr z. B. eine Reise nach Venedig verbot. ${ }^{57}$ Den Auslöser zur Flucht habe schließlich die Drohung ihres Mannes gebildet, sie in der Festung Paliano einzuschließen. ${ }^{58}$

Freiheit ist das eigentliche Leitmotiv der Apologia. Unter diesem Motto verbindet sich Kritik an Lorenzo Onofrio mit einer generelleren Kritik an der restriktiven römischen Gesellschaft. ${ }^{59}$ Maria Mancini motiviert ihre Flucht nicht zuletzt mit ihrem Widerwillen gegen den römischen Lebensstil, der durch Verstellung, Rivalität und Hass geprägt sei:

"Alle jene Gründe, verbunden mit der Aversion, die ich schon immer gegen die italienischen Bräuche und den römischen Lebensstil hegte, wo Täuschung und Hass zwischen den Familien mehr als irgendwo sonst herrschen, zwangen mich dazu, die geplante Flucht nach Frankreich zu beschleunigen. $\ll^{60}$

Ähnlich wie Königin Christina von Schweden, die als Ausländerin in Rom ebenfalls eine Außenseiterposition einnahm, rebellierten sowohl Maria als 
auch Ortensia Mancini gegen die Normen, die in der römischen Gesellschaft für Frauen galten. ${ }^{61}$ Maria bezeichnete Italien als "paese pieno di formalità «, d. h. als ein Land, das quasi in Förmlichkeiten ersticke. ${ }^{62}$ Ortensia schrieb, der Stolz einer römischen Frau bestehe darin, möglichst unauffällig zu bleiben. ${ }^{6}{ }^{3}$ In den apokryphen Memoiren der Maria Mancini heißt es, der Wirkungskreis der römischen Frauen ende an den Fenstervorhängen. ${ }^{64}$ In der Tat war Ortensia dafür kritisiert worden, dass sie sich am Fenster hatte sehen lassen. Da dies in Rom nicht Brauch sei, soll ihre römische Tante sie deswegen sogar aus ihrem Palast geworfen haben. ${ }^{65}$

Erst vor diesem Hintergrund lässt sich ermessen, wie skandalös es war, dass Maria und Ortensia Mancini sich im Sommer 1669 öffentlich in Nymphenverkleidung zeigten. ${ }^{66} 1671$ nahm Maria sich gar die Freiheit heraus, im Tiber zu baden, was von mehreren römischen Avvisi kritisch als unübliche französische Mode kommentiert wurde. ${ }^{67}$ Im Palazzo Colonna wurde eine Festkultur im französischen Stil gepflegt, wozu auch viele opulente Maskeraden zählten. Diese ermöglichten es Maria Mancini, alternative Frauenrollen zu propagieren. Beispielsweise zeigte sie sich in einem überlebensgroßen Bildnis als Circe, die Männer in Affen verzaubern kann (Abb. 4), ${ }^{68}$ oder trat amazonenhaft als die Kriegerin Clorinda auf. ${ }^{69}$

4. Jacob Ferdinand Voet (zugeschrieben): Maria Mancini in orientalisierendem Gewand, um 1670, Öl aufLeinwand. Rom, Palazzo Colonna

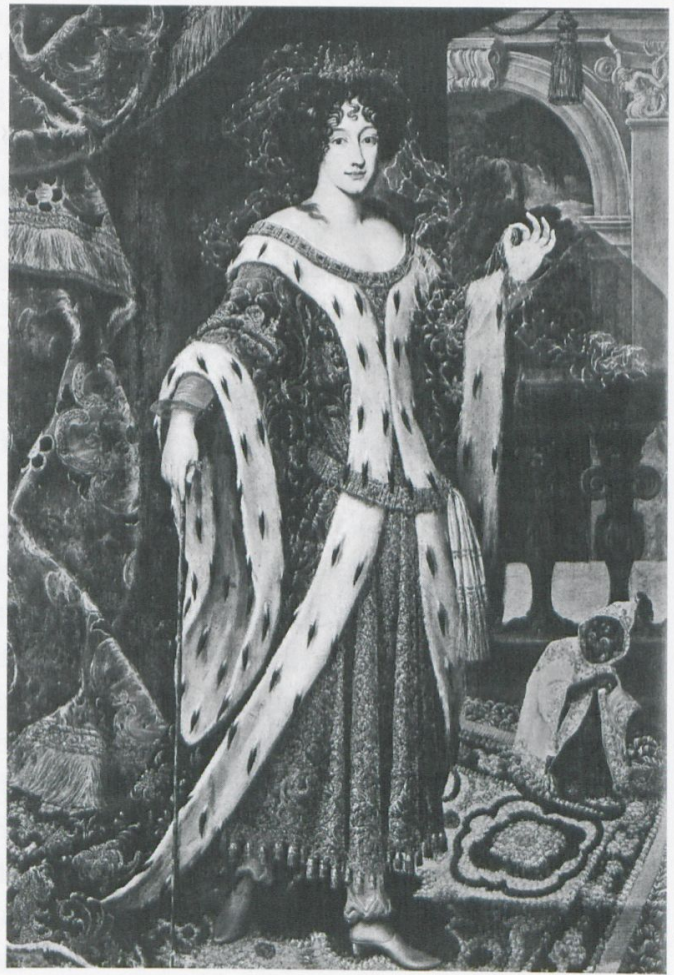




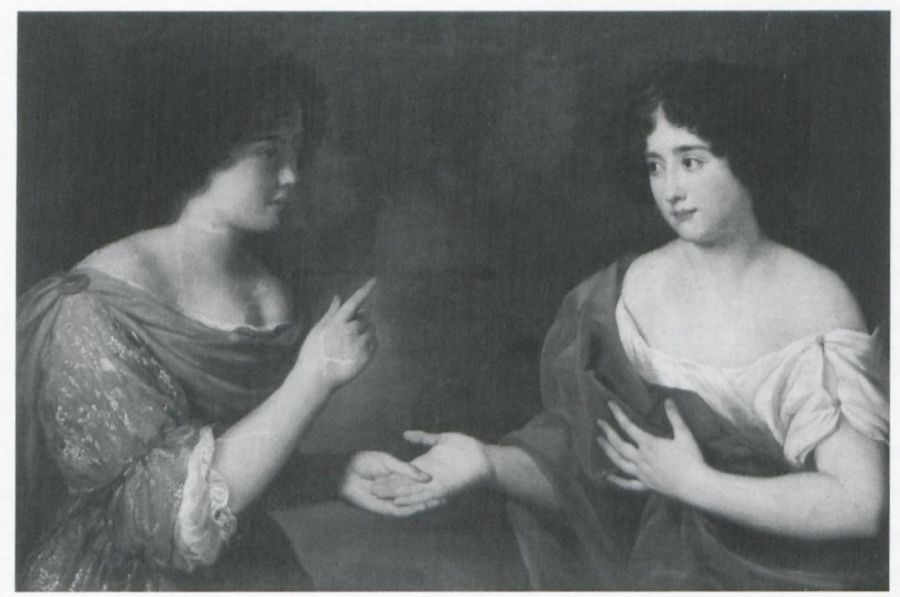

5. Jacob Ferdinand Voet: Maria Mancini liest ihrer Schwester Ortensia aus der Hand, zwischen 1668 und 1672, Öl aufLeinwand, 77,5 ×115 cm. Rom, INPS

Die Clorinda-Maskerade fand auch Erwähnung in der Apologia, da sie der Verteidigung von Marias Reputation diente: »Um mich gegen die üble Nachrede zahlreicher Personen zu wehren, die meinen freien Lebensstil innerhalb eines Landes voller Formalitäten kritisierten, beschloss ich, eine Maskerade zu veranstalten [...]. $\ll^{70}$ Indem Maria sich männlich kostümierte, begegnete sie der Kritik an ihrem und Ortensias »männlichem « Auftreten. ${ }^{71}$ Die Verse, die Maria in der Rolle der Clorinda rezitierte, besagten, dass sie trotz Männerkleidung den Anstand wahre - was man von vielen anderen Frauen nicht behaupten könne, die sich zwar als tugendsame Penelope präsentierten, im Herzen aber eine lasterhafte Phryne seien. ${ }^{72}$

Wie bereits erwähnt, verabscheute Maria Mancini an der römischen Gesellschaft vor allem die »dissimulazione", also die Verstellung. ${ }^{73}$ Im Gegensatz dazu erheben ihre Memoiren den Anspruch, das Erlebte mit größter Wahrhaftigkeit zu schildern. ${ }^{74}$ In einer kurzen literarischen Charakterstudie attestierte Maria sich selbst nicht nur Aufrichtigkeit, sondern auch zahlreiche weitere glanzvolle Eigenschaften: geistige Brillanz, einen weiten Horizont, ein gutes Urteilsvermögen, ein einnehmendes Wesen, Stärke, Mut, Nobilität, Sensibilität und Größe. ${ }^{75}$ Ganze Welten liegen zwischen solchen Äußerungen und den eingangs zitierten Postulaten, römische Frauen sollten sich vor allem mit Handarbeiten beschäftigen! Maria Mancini reklamiert hier für sich Eigenschaften, die in Rom traditionell Männern zugeschrieben wurden - ebenso wie sie sich eine quasi männliche Bewegungsfreiheit herausnahm.

Ein besonders ungewöhnliches Rollenporträt zeigt Maria Mancini als Wahrsagerin, die ihrer Schwester Ortensia aus der Hand liest (Abb. 5). ${ }^{76}$ Aus den Memoiren wissen wir, dass sie sich sowohl mit Astrologie als auch mit 
6. Giuseppe Maria Mitelli: Satirischer Holzschnitt, vor 1660. Mailand, Sammlung Bertarelli

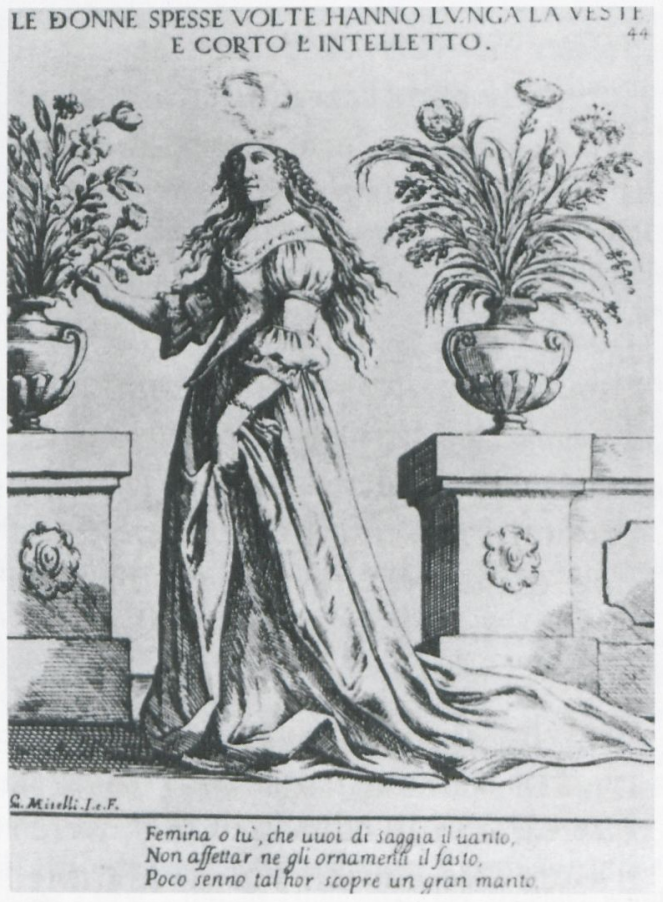

Handlesekunst befasste; ${ }^{77} 1670$ und erneut 1671 veröffentlichte sie sogar einen Discorso astrosofico delle mutationi de' tempi e d'altri accidenti mondani ${ }^{78}$ Die Komposition des Gemäldes von Jacob Ferdinand Voet geht klar auf Caravaggios berühmtes Bild der Wahrsagerin zurück. ${ }^{79}$ Maria Mancini identifizierte sich also einerseits mit einer gesellschaftlichen Außenseiterin, zeigte andererseits aber auch, dass sie sich im Besitz eines privilegierten Geheimwissens fühlte.

Marias Geste in Voets Gemälde dürfte als Ermahnung zu deuten sein. Es geht offenbar nicht darum, ein vorgegebenes Schicksal einfach anzunehmen, sondern im Wissen um bestimmte zukünftige Ereignisse das Handeln darauf abzustimmen. So jedenfalls wird der Schicksalsbegriff in Maria Mancinis Memoiren gebraucht. Die Autorin behauptet zwar, sie werde vom Schicksal verfolgt und geplagt, ${ }^{80}$ resigniert deswegen aber keineswegs. Vielmehr ist die Apologia die Autobiographie einer Frau, die gerade in der schwierigen Zeit nach ihrer Flucht aus Rom nie aufgibt, sondern alles in ihrer Macht Stehende dafür tut, ihre Freiheit zu bewahren. ${ }^{81}$ Dieses Leitmotiv der weiblichen Freiheit macht die Radikalität und besondere Bedeutung von Maria Mancinis Memoiren im Kontext des römischen Seicento aus. 


\section{Schlussbetrachtungen}

Der vorliegende Beitrag hat einen ersten Überblick über theoretische Reflexionen gegeben, die im Rom des Seicento die Frauenrolle zu definieren suchten. Obwohl die Querelle des Femmes vor allem im 16. Jahrhundert mit großer Intensität ausgetragen worden war, war sie im 17. Jahrhundert immer noch hochaktuell: Geradezu programmatisch klagte Maria Mancini im ersten Satz ihrer Apologia, es gelte bei Hofe als amüsante Neckerei, das weibliche Geschlecht herabzusetzen. ${ }^{82}$

Mit Bronzinis Dialog Della dignità, E nobiltà delle donne und Mancinis Apologia standen zwei Texte im Zentrum dieses Aufsatzes, die ungewöhnlich große politische und persönliche Handlungsspielräume für Frauen beanspruchten. Dies wurde weder in der gesellschaftlichen Realität noch in der Sphäre der Literatur geduldet, wie sich an Lorenzo Onofrio Colonnas transeuropäischer »Jagd « nach seiner Frau und den Zensurbestrebungen der Inquisition erkennen lässt. In diesem Kontext kann Flaminio Allegrinis Bildzyklus in der Galleria Deti als misogyn gefärbter Diskussionsbeitrag zur Querelle gelesen werden. Während der Kardinal Deti Eva als femme fatale bzw. Quelle allen Übels präsentierte, konstruierten Jacob Ferdinand Voets Porträts hingegen ein alternatives Frauenbild: Maria Mancini tritt uns darin als starke, selbstbewusste, wissende und Macht ausübende Frau entgegen (Abb. 4, 5).

Wie aus den zitierten Quellen hervorgeht, war die Geringschätzung von Frauen gerade in Rom, der von (meistens) zölibatären Männern dominierten Stadt der Kardinäle, weit verbreitet. Angesichts dessen ist es umso bemerkenswerter, dass Cristofano Bronzini und Maria Mancini für die Anerkennung der weiblichen intellektuellen Eigenständigkeit eintraten. Sie widersetzten sich damit einem populären Klischee, das auch bildlich Verbreitung fand (Abb. 6): Trotz großer Röcke haben die Protagonistinnen von Bronzinis und Mancinis Texten gerade keinen kleinen Verstand $!^{83}$

${ }^{1}$ Giulia Bochi: L'educazione femminile dall' umanesimo alla controriforma. Antologia, Bologna 1961, S. 262: "Si richiede alla giovinetta di buona famiglia di saper recitare l'ufficio della Vergine e dei Santi, e nel rimanente a filare e cucire e occuparsi degli altri esercizi donneschi.«

2 Ibid., S. 265: »io temo che, essendo il sesso femminile per sua natura vano, non ne divenga tanto più altiero $\ll$.

${ }^{3}$ Renata Ago: Maria Spada Veralli, la buona moglie, in: Giulia Calvi (Hrsg.): Barocco al femminile, Rom u. Bari 1992, S. 51-70, S. 52: stutti gli scrittori, li quali trattano del modo del vivere delle dame, anzi delle Principesse e delle Regine, lodano e danno per precetto, che debbano fuggire l'ozio con l'esercitarsi in casa con le loro serve, e donzelle in questi esercizij donneschi di lavorare in lino, e la lana, e la seta, \& altre simili cose $\ll$.

4 Ago 1992, S. 62: "la principessa Borghese è applicata con 60 e più donne e maestri a ricamar in casa le carrozze e livree ed habbito del principe, il quale avrà indosso sopra 100 mila scudi di perle e gioie «.

5 Ago 1992; Marina d'Amelia: Becoming a Mother in the Seventeenth Century. The Experience of a Roman Noblewoman, in: Anne Jacobson Schutte, Thomas Kuhn u. Silvana Seidel 
Menchi (Hrsg.): Time, Space, and Women's Lives in Early Modern Europe, Kirksville 2001, S. 233-244; Carolyn Valone: Matrons and Motives. Why Women Built in Early Modern Rome, in: Sheryl E. Reiss u. David G. Wilkins (Hrsg.): Beyond Isabella . Secular Women Patrons of Art in Renaissance Italy, Kirksville 2001, S. 317-335; Marina d'Amelia: La nuova Agrippina. Olimpia Maidalchini Pamphilj e la tirannia femminile nell'immaginario politico del Seicento, in: Francesca Cantù (Hrsg.): I linguaggi del potere nell'età barocca. Donne e sfera pubblica, Rom 2009, S. 45-96; Marina Caffiero: Sovrane nella Roma dei papi. Cerimoniali femminili, ruoli politici e modelli religiosi, in: ibid., S. 97-124; Katherina A. McIver: An Invisible Enterprise. Women and Domestic Architecture in Early Modern Italy, in: Katherine McIver (Hrsg.): Wives, Widows, Mistresses, and Nuns in Early Modern Italy. Making the Invisible Visible through Art and Patronage, Farnham 2012 , S. 159-177, S. 166-168 u. 170-171 (über römische Auftraggeberinnen).

${ }^{6}$ Simona Feci u. Maria Antonietta Visceglia: Tra due famiglie. Anna Colonna Barberini sprefetessa« di Roma, in: Francesca Cantù (Hrsg.): I linguaggi del potere nell'età barocca. Donne e sfera pubblica, Rom 2009, S. 257-327, S. 282.

7 Anna blieb nicht freiwillig in Rom: Feci u. Visceglia 2009, S. 308.

8 Ibid., S. 299.

${ }_{9}$ Ibid., S. 300 .

${ }^{10}$ Ibid., S. 303: »havendo inteso la disorbitante proposta della sorella, mandò al Senato romano, à farle sapere che egli non hebbe in quella sciocchezza parte alcuna, ma era pronto di assistere alla giusta petitione del Popolo.«

${ }^{11}$ Margarete Zimmermann: Vom Streit der Geschlechter. Die französische und italienische Querelle des Femmes des 15. bis 17. Jahrhunderts, in: Die Galerie der Starken Frauen. Die Heldin in der französischen und italienischen Kunst des 17. Jahrhunderts (hrsg. v. Bettina Baumgärtel u. Silvia Neysters), Ausstellungskatalog, Düsseldorf u. Darmstadt 1995, S. 14-33, S. 21.

12 Margarete Zimmermann: La »Querelle des Femmes « come paradigma culturale, in: Silvana Seidel Menchi, Anne Jacobson Schutte u. Thomas Kuehn (Hrsg.): Tempi e spazi di vita femminile tra medioevo ed età moderna, Bologna 1999, S. 157-173, S. 160 u. 163-166.

13 Zimmermann 1995, S. 15 u. 21-22.
${ }^{14}$ Xenia von Tippelskirch: Die Indexkongregation und die Würde der Frauen: Cristofano Bronzini, "Della dignità e nobiltà della donne«, in: Anne-Marie Bonnet u. Barbara Schellewald (Hrsg.): Frauen in der Frühen Neuzeit. Lebensentwürfe in Kunst und Literatur, Köln, Weimar u. Wien 2004, S. 235-262.

15 Zur Biographie der beiden Frauen siehe Ilaria Hoppe: Maria Magdalena von Österreich (1589-1631). Die vergessene Regentin, in: Christina Strunck (Hrsg.): Die Frauen des Hauses Medici. Politik, Mäzenatentum, Rollenbilder (1512-1743), Petersberg 2011, S. 106-115; Christina Strunck: Christiane von Lothringen, Großherzogin der Toskana (1565-1636), in: ibid., S. 74-93.

${ }^{16}$ Das Porträt ist abgebildet, aber nicht analysiert bei Tippelskirch 2004, S. 239.

${ }_{17}$ Dass die palle das Subjekt der Aussage sind, wird durch die unter dem Porträt angebrachten Verse bestätigt: "Hor ch'il Sol de sei Mondi a me comparte / Con influss(o) benigno i raggi suoi / Ad onta del oblio havran gl'Eroi / Del Femineo Valor Bronzine Carte.« Mit den »sei Mondi ("sechs Welten «) sind wiederum die palle gemeint, die als Himmelskörper gedeutet wurden, seit Galileo Galilei die von ihm entdeckten JupiterMonde nach den Medici benannt hatte. Bronzinis Gedicht besagt, dass die Medici-Gestirne einen wohltätigen Einfluss auf ihn ausüben, was wiederum auf seine Protektion durch die Medici anspielt.

${ }^{18}$ Hierzu ausführlich Strunck 2011, S. 81-83.

${ }^{19}$ Die genannten Kontakte Bronzinis zu Christiane von Lothringen sind dokumentiert in Christina Strunck: Progetti medicei a Roma nei primi decenni del Seicento: dal carteggio della Granduchessa Cristina di Lorena, in: Vincenzo Cazzato, Sebastiano Roberto u. Mario Bevilacqua (Hrsg.): La Festa delle Arti. Scritti in onore di Marcello Fagiolo per cinquant'anni di studi, Rom 2014, Bd. I, S. 452-457, S. 453-455. Eine ausführlichere Analyse von Bronzinis Rolle am Florentiner Hof sowie eine detaillierte Untersuchung der dortigen Auseinandersetzung mit der Querelle des Fermmes findet sich in Christina Strunck: Christiane von Lothringen am Hof der Medici. Geschlechterdiskurs und Kulturtransfer zwischen Florenz, Frankreich und Lothringen (1589-1636), Petersberg 2016 (im Druck).

${ }^{20}$ Robert Valerius: Weibliche Herrschaft im 16. Jahrhundert. Die Regentschaft Elisabeths I. zwi- 
schen Realpolitik, Querelle des femmes und Kult der Virgin Queen, Herbolzheim 2002, S. 187-193; Claudia Opitz: Gleichheit der Geschlechter oder Anarchie? Zum Gleichheitsdiskurs in der Querelle des Femmes und in der politischen Theorie um 1600, in: Gisela Engel, Friederike Hassauer, Brita Rang und Heide Wunder (Hrsg.): Geschlechterstreit am Beginn der europäischen Moderne. Die Querelle des Femmes, Königstein u. Taunus 2004, S. 307-329, S. 315-318.

${ }^{21}$ Margarete Zimmermann: Salon der Autorinnen. Französische dames de lettres vom Mittelalter bis zum 17. Jahrhundert, Berlin 2005, S. 219-221.

${ }^{22}$ Zimmermann 2005, S. 174-183.

23 Elogio della Gran Caterina de Medici, Reina di Francia Madre del Re. Fatto in lingua Italiana, \& Latina, per M. Matteo Zampini, et Tradotto in Francese, per M. Carlo Paschali, et In Spagnuola per l'Tllustre Signor Girolamo Gondi, Gentilhuomo Ordinario della Camera di S. Maestà Christianissima, Paris 1586.

${ }^{24}$ Libro di M. Giovanni Boccaccio Delle Donne Illustri. Tradotto di Latino in Volgare per M. Giuseppe Betussi, con una giunta fatta dal medesimo, D'Altre Donne Famose, e un'altra nuova giunta fatta per $M$. Francesco Serdonati d'altre Donne Illustri Antiche e Moderne, Florenz 1596, S. 526 (»Valdruda Duchessa di Lorena«), 526-527 (»Geltruda di Lorena«), 532-533 (»Doda Duchessa di Loreno«), 633-636 ( Madama Giovanna d'Austria Gran Duchessa di Toscana«) u. 656-659 (»Donna Leonora di Toledo Duchessa di Fiorenza«). Bis 1569 waren die Medici Herzöge von Florenz, danach Großherzöge.

25 Boccaccio/Serdonati 1596, S. 645-655 ("Caterina de Medici Reina di Francia«) u. 671-676 (»Madama Cristiana di Lorena Gran Duchessa di Toscana«).

${ }^{26}$ Ibid., S. 672-673: „ella fu allevata, e ammaestrata da Caterina de Medici Reina di Francia sua avola materna, che fu un vero ritratto di prudenza, e di valore, e un chiaro lume delle donne, e da lei fu amata a dismisura, si che fu compagna di molti suoi viaggi, partecipe di tutti i pensieri, consapevole di tutti i segreti, di tutte le imprese, e di tutte le cure: onde ella apprese la cognizione di tutte le cose appartenenti al reggere stati, e governar popoli.«

${ }^{27}$ Ibid., S. 479-490.

28 Discorsi Sopra Cornelio Tacito del Conte Virgilio Malvezzi. Al Serenissimo Ferdinando II. Gran Duca di Toscana, Venedig 1622, S. 180: "non e cosa ne più odiosa, ne più aborrita da gli huomini, che il servire ad una Donna, come repugnante alla ragione, contrario a' precetti di Dio, e contrarijssimo alle leggi della natura. [...] per essere quel sesso (non dico sempre, ma il più delle volte) privo di prudenza, e di valore; con molta superbia, ed altretanta libidine, e per conseguenza inettissima a governare. Wie aus dem Widmungstext hervorgeht, stand Malvezzi in einem bereits etablierten Klientelverhältnis zum Medici-Hof.

29 Della Dignità, \& Nobiltà delle Donne. Dialogo di Cristofano Bronzini d'Ancona. Diviso in quattro Settimane; e ciascheduna di esse in Sei Giornate. Alla Serenissima Arciduchessa d'Austria Maria Maddalena, Gran Duchessa di Toscana. Settimana Prima, e Giornata Prima, Florenz 1622, S. 57-73.

${ }^{30}$ Della Virtù, e Valore delle Donne Illustri. Dialogo di Christofano Bronzini d'Ancona. Diviso in quattro Settimane; e ciascheduna di esse in sei Giornate. Settimana Seconda, Giornata Settima, Florenz 1632, S. 62-78. Die Aussage, Christiane könne sowohl Frauen als auch Männern als Vorbild dienen, findet sich auf S. 78. Textimmanente Hinweise deuten darauf hin, dass dieser Text bereits vor 1624 verfasst wurde, da Lucrezia Marinella ihn in De' Gesti Heroici e della Vita Maravigliosa della Serafica S. Caterina da Siena (Venedig 1624) rezipierte. Hierzu ausführlich Strunck 2016 (vgl. Anm. 19).

31 Tippelskirch 2004, S. 257.

32 Tippelskirch 2004, S. 249, Anm. 50: »essersi nella Donna terminata et haver havuto fine tutta la sapienza et potenza del summo facituore, come dignissima sopra tutte le altre creature, dopo la quale non restò a Dio di creare cosa più eccellente e più degna di lei, oltre della quale altra creatura non si trova ne immaginar si puote.«

33 Tippelskirch 2004, S. 249, Anm. 50: »esser stata da Dio creata la Donna signora dell'huomo, et l'huomo a lei soggetta, et per servir lei, benche per il peccato poi ne fusse al'huomo fatta sogetta«.

${ }^{34}$ Maria Cristina Guardata: Flaminio Allegrini in Palazzo Chigi, in: Claudio Strinati u. Rossella Vodret (Hrsg.): Palazzo Chigi, Mailand 2001, S. 131-143, S. 131.

${ }^{35}$ Giovanni Battista Agucchis Beschreibung des Bildprogramms der Villa Aldobrandini in Frascati findet sich abgedruckt bei Cesare D'Onofrio: La Villa Aldobrandini di Frascati, Rom 1963, S. 97-99. 
${ }^{36}$ Luigi Devoti: La Villa Belvedere Aldobrandini di Frascati, Velletri 1990, S. 74; Guardata 2001, S. 131 u. 133.

37 D'Onofrio 1963, Abb. 18-20; Devoti 1990, Abb. S. 83; Guardata 2001, Abb. S. 138-140.

38 D'Onofrio 1963, Abb. 21-24; Devoti 1990, Abb. S. $78,81,86,87$.

39 Sigrid Epp: »La femina origine di ogni male«. Affreschi nella Galleria Deti del Palazzo Chigi a Roma, in: Studi di storia dell'arte 12, 2001, S. $75-94$, S. 87.

40 Epp 2001, Abb. 12 u. 13.

41 Epp 2001, S. 78, führt Belege dafür an, dass die generell positiv bewerteten Handlungen von Judith und Jael im 17. Jahrhundert durchaus auch Kritik auf sich zogen.

42 Sam 13,1-21.

${ }^{43}$ Ri 19, 1-30.

${ }^{44} \mathrm{Zu}$ den zwei genannten Episoden vgl. Epp 2001, S. 79.

${ }^{45}$ Die beiden bislang publizierten Interpretationen des Bildzyklus sind einander genau entgegengesetzt: Epp 2001 liest darin eine misogyne Botschaft, während Guardata 2001, S. 135, betont: "This cycle's intent is not misogynous. [...] This cycle represents a vision of Sin and the Redemption of mankind by women.« Der Umstand, dass beide Lesarten möglich sind, spricht meiner Meinung nach für eine intendierte, zur Debatte anregende Ambivalenz.

${ }^{46}$ Epp 2001, S. 76; Guardata 2001, S. 134.

47 Zum folgenden ausführlicher Dizionario biografico degli italiani, Bd. 68, Rom 2007, S. 521-527, s. v. »Mancini, Maria (Stefano Tabacchi); Claude Dulong: Marie Mancini. La première passion de Louis XIV, Paris 1993; Christina Strunck: Libertins in Verkleidung. Die Pariser Précieuses, Maria Mancini und die römische Orientfaszination des Seicento, in: Eckhard Leuschner u. Thomas Wünsch (Hrsg.): Das Bild des Feindes. Konstruktionen von Antagonismen und Kulturtransfer im Zeitalter der Türkenkriege. Ostmitteleuropa, Italien und Osmanisches Reich, Berlin 2013, S. 471-494, S. 479484.

${ }^{48}$ Siehe dazu insbesondere Elisabetta Graziosi: Lettere da un matrimonio fallito. Maria Mancini al marito Lorenzo Onofrio Colonna, in: Gabriella Zarri (Hrsg.): Per lettera. La scrittura epistolare femminile tra archivio e tipografia, secoli XVXVII, Rom 1999, S. 535-587.
49 Dulong 1993, S. 157-163; Kirsten Beckmann: Inszenierter Skandal als Apologie? Die Memoiren der Hortense und Marie Mancini, Diss. Trier 2004, S. 104-111, URL: http://ubt.opus.hbz-nrw.de/volltexte/ 2004/269/; Francesco Petrucci: Ferdinand Voet (1639-1689) detto Ferdinando de' Ritratti, Rom 2005, S. 13-14.

50 Petrucci 2005, S. 13-14, 43 u. 190-193.

51 Maria Mancini: Apologia, ovvero le autentiche memorie di Maria Mancini connestabile Colonna, scritte da lei stessa, in: idem.: I dispiaceri del Cardinale (hrsg. v. Daria Galateria), 2. Aufl. Palermo 1991, S. 9-163, S. 57-58; Dulong 1993, S. 160 u. 163; Beckmann 2004, S. 92, 106-107 u. 117.

52 Hierzu und zum Folgenden Graziosi 1999, S. 574-577; Beckmann 2004, S. 36-38, 45-133, speziell S. 109-111.

53 Beckmann 2004, S. 36-41 u. 278-284.

54 Mancini 1991, S. 41-49.

55 Eduard A. Safarik: Palazzo Colonna, Rom 1999, S. 240 u. 242 (mit Abb.).

56 Mancini 1991, S. 67-68; vgl. Graziosi 1999, S. 546 .

57 Mancini 1991, S. 55; vgl. Graziosi 1999, S. 547 .

58 Mancini 1991, S. 68; vgl. Graziosi 1999, S. 544-545.

${ }^{59} \mathrm{Zu}$ Beginn der Ehe sei diese Abneigung gegen den römischen Lebensstil noch durch die Liebe zu Lorenzo Onofrio Colonna ausgeglichen worden: "Quantunque le usanze italiane non fossero troppo di mio gusto, l'amore che provavo già per il Connestabile me le rendeva piacevoli.« (Mancini 1991, S. 41.)

${ }^{60}$ Mancini 1991, S. 69: "Tutte queste ragioni, unite all'avversione che avevo sempre avuto per i costumi italiani, e per il modo di vivere di Roma, dove la dissimulazione e l'odio tra famiglie regnano più sovranamente che in qualunque altra parte, mi costrinsero ad affrettare l'esecuzione del progetto [...] di rifugiarmi in Francia.«

${ }^{61} \mathrm{Zu}$ Christina von Schweden vgl. den Beitrag von Veronica Biermann im vorliegenden Band. Auf Bitten Maria Mancinis versprach Christina von Schweden, Ortensia in einer schwierigen Situation Zuflucht zu gewähren, hielt sich letztlich aber nicht daran: Mancini 1991, S. 59.

${ }^{62}$ Mancini 1991, S. 53.

${ }^{63}$ Elena Tamburini: Due teatri per il Principe. Studi sulla committenza teatrale di Lorenzo Onofrio 
Colonna (1659-1689). Con un'ipotesi di ricostruzione del teatro "piccolo" elaborata in collaborazione con Sergio Rotondi, Rom 1997, S. 43.

${ }_{64}$ Tamburini 1997, S. 43.

65 Beckmann 2004, S. 108.

${ }_{66}$ Tamburini 1997, S. 113.

67 Tamburini 1997, S. 123. Zu Marias »französischem» Lebensstil vgl. auch Graziosi 1999, S. 548 .

${ }^{68}$ Zur Ikonographie dieses Porträts: Strunck 2013, S. 481-482.

69 Tamburini 1997, S. 110-111.

${ }^{70}$ Mancini 1991, S. 53: "per ribattere alla maldicenza di molti che trovavano da ridire sul mio modo di vivere libero in un paese pieno di formalità decisi di fare una mascherata nella quale, sotto forma di Clorinda [...] andavo recitando i versi $[\ldots]$ :

\section{D'obliato decoro}

Questo amante guerrier non dia sospetto

Che s'ho viril aspetto

Intatto d'onestà serbo il tesoro;

Quante in ogni confine

Son Penelope al volto, al cor, son Frine.«

${ }^{71}$ Über Maria Mancini wurde berichtet, sie gehe wie ein Mann und trage »con una libertà francese ( (mit französischer Freiheit») kurz geschnittene Haare, Tamburini 1997, S. 46, Anm. 81. Ortensia Mancini wurde für das Tragen von Männerkleidung gerügt, Beckmann 2004, S. 117.

72 Siehe oben Anm. 70.

73 Mancini 1991, S. 69.

74 Dies wird schon im Titel der französischen Erstausgabe von 1676 deutlich: La Verité dans son jour ou les véritables memoires de Marie Mancini, connétable Colonne. Die 1678 in Leiden und 1679 in Köln erschienene überarbeitete Fassung trug ebenfalls die Wahrheit im Titel: Apologie ou les véritables mémoires de Madame Marie Mancini, connétable de Colonna, écrits par elle-même (Beckmann 2004, S. 39-40).

${ }^{75}$ Dulong 1993, S. 166: „Ce corps est animé d'un esprit de grande étendue, vif, net, délicat, subtil, ardent et brillant [...]; il est pourtant soutenu d'un jugement ferme, clair et juste [...].« Maria besitze »un certain air aisé et des manières naturellement engageantes [...]; et cela suit le caractère d'une âme comme est la mienne, ferme, intrépide, noble, sensible, grande, sincère et toujours bienfaisante.

${ }^{76}$ Petrucci 2005, S. 28 u. 190.

77 Tamburini 1997, S. 44. Siehe auch Beckmann 2004, S. 41 (Brief Mazarins über Maries astrologische »Lehrstunden « bei einem Araber).

78 Tamburini 1997, S. 45.

${ }^{79}$ Giorgio Bonsanti: Caravaggio, Florenz 1984, S. 13.

80 Mancini 1991, S. 102-103.

${ }^{81}$ Mancini 1991, S. 74-135; Graziosi 1999, S. 548-553 u. 562-567.

${ }^{82}$ Mancini 1991, S. 11.

${ }^{83}$ Auf Mitellis Graphik (Abb. 6) verwies Mary D. Garrard: Artemisia Gentileschi. The Image of the Female Hero in Italian Baroque Art, Princeton 1989, S. 356-357. Die Inschrift lautet wörtlich übersetzt: "Die Frauen haben oft ein langes Gewand und einen kurzen Intellekt.« 